\title{
Prognostic markers of recurrence and survival in rectal cancer treated with neoadjuvant chemoradiotherapy and surgery
}

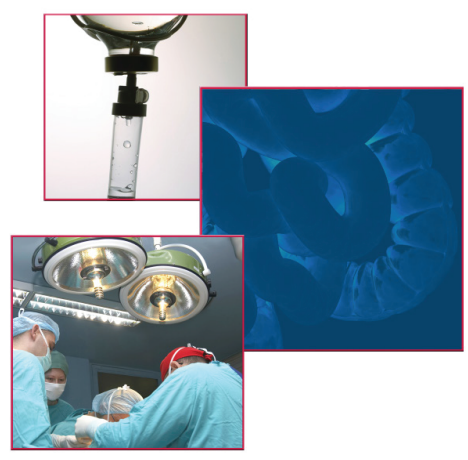

Michael Abdalmassih*,1, Gokulan Sivananthan ${ }^{1}$, Zachary Raizman ${ }^{1}$, Pascal Lambert ${ }^{2}$, Debrah Wirtzfeld ${ }^{3}$, Bashir Bashir ${ }^{1,4}$, Robert Wightman ${ }^{5}$, Kroeker Daniel ${ }^{3}$ \& Maged Nashed ${ }^{1,4}$

${ }^{1}$ Cancer Care Manitoba, Radiation Oncology, 675 McDermot Ave, Winnipeg, MB, R3E 0V9, Canada

${ }^{2}$ Cancer Care Manitoba, Epidemiology \& Cancer Registry, Winnipeg, MB, Canada

${ }^{3}$ Department of Family Medicine, University of Manitoba, Winnipeg, MB, R3T 2N2, Canada

${ }^{4}$ Department of Radiology, University of Manitoba, Radiology, Winnipeg, MB, Canada

${ }^{5}$ Department of Pathology, University of Manitoba, Pathology, Winnipeg, MB, Canada

*Author for correspondence: mnashed1@CancerCare.mb.ca

\section{Practice points}

- In our cohort of patients with locally advanced rectal cancer who received neoadjuvant chemoradiotherapy and surgery.

- The rate of pathological complete response (pCR) was $18.9 \%$.

- The 5-year rate of recurrence was $33.2 \%$.

- The 5-year overall survival rate was $77 \%$.

- Lymphovascular invasion in pathological specimen was associated with recurrence.

- pCR was associated with less recurrence and better survival and should be considered as an oncological end point.

- Aduvant chemotherapy was associated with better survival.

- The patients who achieved pCR may not benefit from aduvant chemotherapy.

Aim: To identify markers of recurrence and survival in patients with locally advanced rectal cancer who received neoadjuvant chemoradiotherapy and surgery. Materials \& methods: A total of 280 patients were identified in Manitoba between 2007 and 2012. Demographics and clinical data were collected. Cox regression models were used to identify outcome predictors. Results: A total of 53 patients achieved pathological complete response ( $p C R$ ) and 160 patients received adjuvant chemotherapy (ACT). The median follow-up duration was 2.06 years. Recurrence and survival rates at 5 years were 33.2 and $77.0 \%$, respectively. pCR and lymphovascular invasion predicted recurrence. PCR and ACT predicted better survival. Conclusion: $\mathrm{pCR}$ is a significant predictor of recurrence and survival and may be considered as an oncological end point. The patients who achieve $\mathrm{PCR}$ may not derive additional survival benefit from ACT.

First draft submitted: 1 October 2017; Accepted for publication: 9 February 2018; Published online: 2 May 2018

Keywords: cancer $\bullet \mathrm{LVI} \bullet$ neoadjuvant $\bullet \mathrm{pCR} \bullet$ predictor $\bullet$ rectal $\bullet$ recurrence $\bullet$ survival

Locally advanced rectal cancer (LARC) is defined as the presence of either T3-4 and/or node-positive disease in absence of distant metastases. Neoadjuvant chemoradiotherapy (NACRT) followed by radical surgery that includes total mesorectal excision is standard treatment for these patients $[1,2]$. The estimated 5-year overall survival (OS) and local recurrence (LR) rates were $76 \%$ and $6 \%$, respectively, with the distant recurrence (DR) rate at $36 \%$ in CAO/ARO/AIO-94 study [2]. Similar results were reported by the European Organization for Research and Treatment of Cancer (EORTC) Radiotherapy Group Trial 22921 [1] with a 5-year cumulative incidence of LR at $7.6 \%$ and $\mathrm{DR}$ at $34.4 \%$. 
Recent evidence suggests that the patients with LARC who achieved pathological complete response (pCR) after NACRT have a more favorable long-term outcome compared with patients with incomplete pathologic response. A recent meta-analysis of 3105 patients demonstrated that patients who achieved a pCR had better 5-year OS compared with those who did not ( 83 vs 66\%). The 5 -year DR-free survival was also higher ( 89 vs $75 \%$ ) [3].

Patients with LARC may also receive adjuvant chemotherapy (ACT) as an extrapolation from large randomized controlled trials on colon cancer but it is still debatable if patients derive the same benefit from this approach as patients with colon cancer. For instance, the EORTC 22921 randomized trial showed no survival benefit from ACT after NACRT [4]. Nevertheless, the current guidelines of the National Comprehensive Cancer Network recommend ACT for completely resected stage II and III rectal cancer [5]. The lack of clear evidence of benefit from ACT is presented in the new European Society for Medical Oncology (ESMO) Clinical Practice guidelines [6]. It is reasonable to consider ACT for a subgroup of patients with rectal cancer after NACRT and surgery with the caveat that the level of scientific evidence for benefit is much lower than in colon cancer. For patients who achieve pCR after NACRT, the survival benefit from ACT is likely to be minimal or absent. This was shown in the updated analysis by Maas et al. [7] where those who achieved PCR after NACRT did not derive survival benefit from further chemotherapy postoperatively. This argues that pCR should be considered as an oncological endpoint and strategies to increase the likelihood of achieving pCR should be explored and implemented.

This retrospective study aims to identify the prognostic markers for recurrence and survival in LARC after NACRT and radical surgery.

\section{Methods}

\section{Study population \& data accrual}

This retrospective chart review study was conducted on the patients who diagnosed with rectal adenocarcinoma in the province of Manitoba, Canada between January 2007 and December 2012. Patients were included in the study if they received NACRT followed by radical surgery with a pathology report available for review. The study was approved by the regulatory Local Ethics Committee. Patients were identified through the Manitoba Cancer Registry, which contains information regarding all Manitobans diagnosed with cancer since 1956 [8]. The Manitoba Cancer Registry has among the highest levels of cancer reporting completeness in North America [9].

Initial tumor, node metastasis (TNM) staging was determined based on imaging studies including rectal MRI and/or transrectal ultrasound and contrast-enhanced computed tomography of chest, abdomen and pelvis computed tomography, MRI. Overall staging was determined according to the American Joint Committee on Cancer 7 th Edition guidelines [10]. The patients received neoadjuvant radiation therapy to the pelvis (45-50.4 Gy in 25-28 fractions) with a concurrent 5FU-based chemotherapy. Radiation therapy planning was based on clinical target volumes as described by the radiation therapy oncology group [11]. The patients underwent a total mesorectal excision-based surgery at a median of 56 days (Q1-Q3 43-56 days) post-NACRT. pCR after NACRT was defined as complete disappearance of mural and nodal tumor from the resected specimen as per the protocol of the College of American Pathologists [12].

Data collected included patients' demographics, preoperative staging, NACRT details, type and date of surgery, pathological details, ACT and follow-up. Data collection was completed on 31 August 2016.

\section{Statistical analyses}

Duration of follow-up was calculated as the time from surgery to the event of interest. Patients without event were censored at the date of last follow-up. Time to recurrence was calculated from date of surgery to first event (any recurrence). OS was calculated from date of surgery to date of death. The patients were censored at last follow-up. A Kaplan-Meier curve was used to describe OS rates and cumulative incidence was used to describe recurrence rates. Time-varying Cox regression was used to predict OS and time-varying competing risk regression was used to predict recurrence (death without recurrence as the competing risk). ACT was included as a time-varying predictor. Restricted cubic splines were used to test for the assumption of linearity. Schoenfeld residual plots were used to test for proportional hazard assumption. Influence plots were used to detect influential outliers. Likelihood ratio testing was used for model building. All regression models were run using STATA 14.2 (Stata Corp. LLC, TX, USA).

A total of $8 \%$ of the cohort had missing lymphovascular invasion (LVI) values. That missing data were imputed using the mice package in $\mathrm{R}$ foundation for statistical computing (Vienna, Austria). 30 imputations were produced and verified by comparing the distributions of observed and imputed data conditional on propensity score. Analyses for the cohort were run with complete cases and with the imputed values as a sensitivity analysis. 


\begin{tabular}{|c|c|c|}
\hline Variable & $n$ & $\%$ \\
\hline Age, mean (SD) & 61.18 & (10.91) \\
\hline - Male & 186 & 66.43 \\
\hline - Female & 94 & 33.57 \\
\hline \multicolumn{3}{|l|}{ Residence: } \\
\hline - Rural & 108 & 38.57 \\
\hline \multicolumn{3}{|l|}{ Overall stage: } \\
\hline-11 & 82 & 29.29 \\
\hline- III & 198 & 70.71 \\
\hline \multicolumn{3}{|l|}{ Distance from anal verge $(\mathrm{cm})$ : } \\
\hline - Missing & 3 & 1.07 \\
\hline \multicolumn{3}{|l|}{ Pretreatment CEA $(\mathrm{ng} / \mathrm{ml})$ : } \\
\hline$-\leq 5$ & 162 & 57.86 \\
\hline$->5$ & 94 & 33.57 \\
\hline - Missing & 24 & 8.57 \\
\hline \multicolumn{3}{|l|}{ Surgery type: } \\
\hline - Low anterior resection & 157 & 56.07 \\
\hline - Abdominoperineal resection & 108 & 38.57 \\
\hline - Pelvic exenteration & 6 & 2.14 \\
\hline - Other & 9 & 3.21 \\
\hline
\end{tabular}

\section{Results}

A total of 280 patients were included who met the inclusion criteria with a mean age of 61.18 years. Two-thirds of the study cohort (186) were males. A total of $172(61.43 \%)$ patients resided in the provincial capital (Winnipeg), and $82(29.39 \%)$ and $198(70.71 \%)$ patients had clinical stage II and III rectal cancer, respectively. A total of 34 (12.1\%) patients had an upper rectal cancer while $121(43.2 \%)$ and $122(43.6 \%)$ had mid- and lower-rectal cancers, respectively. A total of 157 (56.07\%) patients underwent low anterior resection while 108 (38.57\%) underwent abdominoperineal resection. Postoperatively, 160 patients (57.14\%) received 5FU-based ACT (Table 1).

Complete pathological response was achieved in 53 patients $(18.62 \%)$ and complete resection (R0) was achieved in $259(92.5 \%)$ patients (Table 2). At a median follow-up of 2.06 years (Q1-Q3 are 0.83-4.02), 68 patients developed relapse (locoregional, distant or both) and 69 patients died.

Although the 5 year rate of LR was low at 4.7\%, the 5 year rate of overall recurrence was $33.2 \%$ (Figure 1 ). pCR was associated with less recurrence (subhazard ration [SHR]: 0.29; 95\% CI: 0.11-0.83; p < 0.02) while LVI detected in surgical pathology was associated with a significantly higher risk of recurrence (SHR: $2.113 ; 95 \%$ CI: 1.19-3.77; $\mathrm{p}<0.011$; Table 3).

The 5-year OS rate was 77\% (Figure 2). pCR and ACT (Table 4) predicted better survival (hazard ratio [HR]: 0.19; 95\% CI: 0.07-0.54; $p<0.002$ and HR: 0.54; 95\% CI: 0.33-0.88; $p<0.013$, respectively). Other predictors of survival included margin status (R1 HR: 4.98; 95\% CI: 2.39-10.38; $\mathrm{p}<0.001$ ), disease stage (stage III HR: 2.279; 95\% CI: 1.26-4.13; p < 0.007), gender (male HR: 1.812; 95\% CI: 1.06-3.12; p < 0.031) and residence (Winnipeg HR: 2.19; 95\% CI: 1.28-3.79; $\mathrm{p}<0.005$ ). 


\begin{tabular}{|c|c|c|}
\hline Variable & $\mathrm{n}$ & $\%$ \\
\hline \multicolumn{3}{|l|}{ pCR: } \\
\hline- No & 227 & 81.07 \\
\hline \multicolumn{3}{|c|}{ Surgical margin status: } \\
\hline$-\mathrm{RO}$ & 259 & 92.50 \\
\hline - Missing & 6 & 2.14 \\
\hline \multicolumn{3}{|l|}{ LVI: } \\
\hline - Yes & 29 & 10.36 \\
\hline- No & 228 & 81.43 \\
\hline - Missing & 23 & 8.21 \\
\hline
\end{tabular}

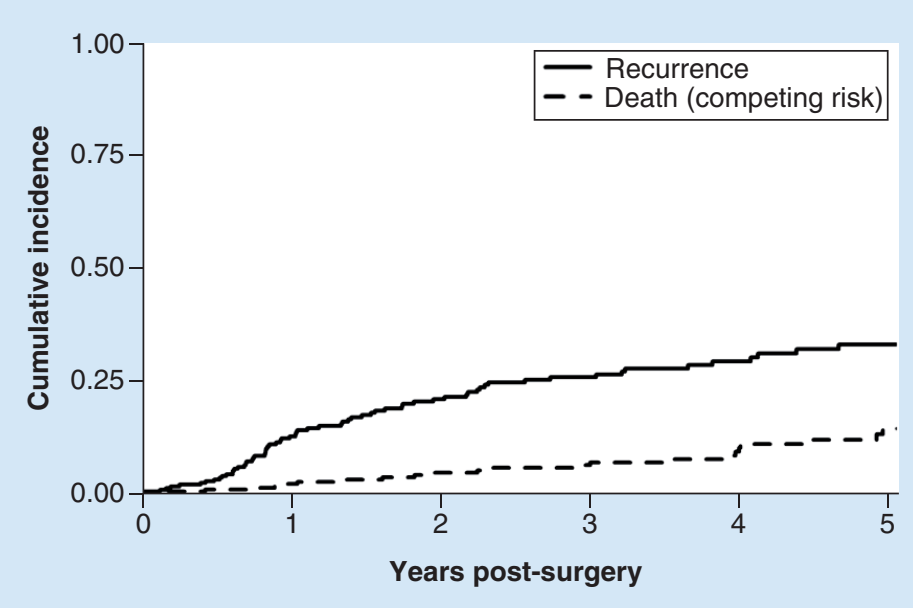

Figure 1. Cumulative incidence of recurrence.

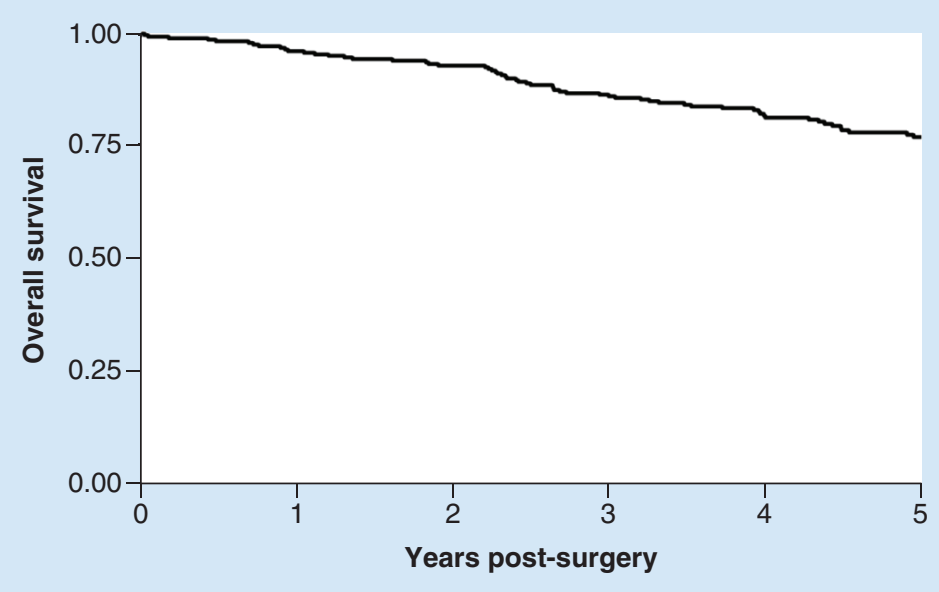

Figure 2. Kaplan-Meier curve of overall survival. 


\begin{tabular}{|c|c|c|c|c|c|c|c|c|c|c|c|c|}
\hline \multirow[t]{2}{*}{ Variable } & \multicolumn{3}{|c|}{ Univariable } & \multicolumn{3}{|c|}{ Univariable (imputed) } & \multicolumn{3}{|c|}{ Multivariable } & \multicolumn{3}{|c|}{ Multivariable (imputed) } \\
\hline & SHR & $95 \% \mathrm{Cl}$ & $p$-value & SHR & $95 \% \mathrm{Cl}$ & $\mathrm{p}$-value & SHR & $95 \% \mathrm{Cl}$ & $\mathrm{p}$-value & SHR & $95 \% \mathrm{Cl}$ & p-value \\
\hline \multicolumn{13}{|l|}{ Gender: } \\
\hline - Male & 0.71 & $0.44-1.16$ & 0.17 & - & - & - & - & - & - & - & - & - \\
\hline - & 1.17 & $0.72-1.93$ & 0.52 & - & - & - & - & - & - & - & - & - \\
\hline - Rural & 1 & - & - & - & - & - & - & - & - & - & - & - \\
\hline \multicolumn{13}{|l|}{ Stage: } \\
\hline$-\mathrm{III} / \mathrm{IV}$ & 1.17 & $0.70-1.96$ & 0.54 & - & - & - & - & - & - & - & - & - \\
\hline - No & 1 & - & - & - & - & - & 1 & - & - & 1 & - & - \\
\hline \multicolumn{13}{|l|}{ LVI: } \\
\hline - Yes & 2.42 & $1.36-4.32$ & 0.003 & 2 & $1.28-4.14$ & 0.005 & 2.11 & $1.19-3.77$ & 0.011 & 2.03 & $1.13-3.66$ & 0.018 \\
\hline - No & 1 & - & - & 1 & - & - & 1 & - & - & 1 & - & - \\
\hline \multicolumn{13}{|l|}{ ACT: } \\
\hline - Yes & 1.11 & $0.68-1.81$ & 0.67 & - & - & - & - & - & - & - & - & - \\
\hline - No & 1 & - & - & - & - & - & - & - & - & - & - & - \\
\hline \multicolumn{13}{|c|}{ Surgical resection status: } \\
\hline$-\mathrm{R} 1$ & 1.89 & $0.83-4.34$ & 0.13 & - & - & - & - & - & - & - & - & - \\
\hline$-R_{0}$ & 1 & - & - & - & - & - & - & - & - & - & - & - \\
\hline
\end{tabular}

\section{Discussion}

In the current study of patients with LARC who were treated with NACRT followed by surgery, pCR was achieved in $18.93 \%$ of the cohort. This rate is higher than the $8 \%$ rate reported in the prospective Phase III trial $\mathrm{CAO} / \mathrm{ARO} / \mathrm{AIO}-94$ study [2]. This difference could be explained by using continuous $5 \mathrm{FU}$ through the full RT course in our study rather than only in the first and final weeks as used in the previously mentioned trial. Other centers in Canada have reported a pCR rate of $18.2 \%$ which is similar to ours [13].

It has been reported that patients who achieve pCR tend to have better long-term outcomes [14-17]. Our results demonstrate that $\mathrm{pCR}$ was a predictor of less recurrence and better survival rates. The $\mathrm{pCR}$ might be an indicator of a tumor with a better biological profile that is reflected as having less recurrences and better survival [3].

Because of the desirable effect of pCR on recurrence and survival, different strategies have been tried to maximize the chance of achieving it. Intensification of chemotherapy as a part of NACRT was tested in many trials [18-21]. Only the CAO/ARO/AIO-04 [21] showed an improvement in the rate of $\mathrm{pCR}$ with intensification of neoadjuvant chemotherapy. Due to lack of convincing evidence, the use of chemotherapy dose-intensification has not been practiced as a part of the routine preoperative NACRT. The use of targeted therapy has also been tried as a part of neoadjuvant therapy. Nevertheless, at present there are no solid and mature data available to demonstrate a possible beneficial effect of adding targeted therapies to the NACRT [22].

The value of escalating the radiation dose was addressed in a non-randomized comparative effectiveness analysis on 3298 patients with rectal cancer [23]. Patients who received higher radiotherapy doses were more likely to be downstaged from cT3-T4 and/or node positive disease to pCR after NACRT. Escalation of radiation dose with intensification of chemotherapy was also tested in the ACCORD 12 trial [24]. A significant increase in complete or near complete response was observed with the neoadjuvant regimen of 50 Gy plus capecitabine/oxaliplatin compared with 45 Gy plus capecitabine. However, the difference in pCR rates of 19.2 versus $13.9 \%$ did not reach statistical significance $(\mathrm{p}=0.09)$. In different prospective trials $[18,19,25], \mathrm{pCR}$ rates did not improve with 


\begin{tabular}{|c|c|c|c|c|c|c|c|c|c|}
\hline \multirow[t]{2}{*}{ Variable } & \multicolumn{3}{|c|}{ Univariable } & \multicolumn{3}{|c|}{ Univariable (imputed) } & \multicolumn{3}{|c|}{ Multivariable } \\
\hline & HR & $95 \% \mathrm{Cl}$ & p-value & HR & $95 \% \mathrm{Cl}$ & $\mathrm{p}$-value & HR & $95 \% \mathrm{Cl}$ & p-value \\
\hline $\begin{array}{l}\text { Age (per } \\
10 \text { years) }\end{array}$ & 1.435 & $1.15-1.79$ & 0.002 & - & - & - & - & - & - \\
\hline \multicolumn{10}{|l|}{ Gender: } \\
\hline - Male & 1.525 & $0.89-2.61$ & 0.124 & - & - & - & 1.812 & $1.06-3.12$ & 0.031 \\
\hline - Female & 1 & - & - & - & - & - & 1 & - & - \\
\hline \multicolumn{10}{|l|}{ Residence: } \\
\hline - Winnipeg & 1.474 & $0.89-2.45$ & 0.134 & - & - & - & 2.199 & $1.28-3.79$ & 0.005 \\
\hline -Rural & 1 & - & - & - & - & - & 1 & - & - \\
\hline \multicolumn{10}{|l|}{ Stage: } \\
\hline$-\mathrm{III} / \mathrm{IV}$ & 1.860 & $1.03-3.35$ & 0.038 & - & - & - & 2.279 & $1.26-4.13$ & 0.007 \\
\hline$-1 / I I$ & 1 & - & - & - & - & - & 1 & - & - \\
\hline \multicolumn{10}{|l|}{ pCR: } \\
\hline - Yes & 0.221 & $0.08-0.61$ & 0.003 & - & - & - & 0.195 & $0.07-0.54$ & 0.002 \\
\hline- No & 1 & - & - & - & - & - & 1 & - & - \\
\hline \multicolumn{10}{|l|}{ LVI: } \\
\hline - Yes & 2.201 & $1.17-4.15$ & 0.015 & 2.070 & $1.10-3.88$ & 0.023 & - & - & - \\
\hline - No & 1 & - & - & 1 & - & - & - & - & - \\
\hline \multicolumn{10}{|l|}{ ACT: } \\
\hline - Yes & 0.674 & $0.42-1.09$ & 0.107 & - & - & - & 0.537 & $0.33-0.88$ & 0.013 \\
\hline- No & 1 & - & - & - & - & - & 1 & - & - \\
\hline \multicolumn{10}{|c|}{ Surgical resection status: } \\
\hline$-\mathrm{R} 1$ & 4.268 & $2.17-8.39$ & $<0.001$ & - & - & - & 4.981 & $2.39-10.38$ & $<0.001$ \\
\hline- Ro & 1 & - & - & - & - & - & 1 & - & - \\
\hline
\end{tabular}

the addition of oxaliplatin to the NACRT without the escalation of the radiation dose. Therefore, this suggests that radiation dose escalation, rather than intensification of chemotherapy, was responsible for the improvement in response rates observed in ACCORD 12 trial.

In our study, LVI was identified as a significant predictor of recurrence. LVI was previously reported to be a predictor of recurrence in early-stage rectal cancer [26-28], which is consistent with our findings. LVI could be detected in the staging MRI with high specificity and moderate sensitivity $[29,30]$ and could potentially be used to direct intensification of NACRT.

The estimated 5 -year OS rate in this report was $77 \%$. This is comparable to what was reported in the CAO/ARO/AIO-94 Phase III trial (76\%) [31]. In Manitoba, the 5-year survival for a cohort of patients with stage I-III rectal cancer who were treated with curative intent between 2004 and 2006 was $71.1 \%$ [32]. The improved survival in our study, in spite of excluding stage I, may be explained by the wide-scale implementation of NACRT in Manitoba in 2007 and the use of oxaliplatin-based ACT.

There is inconsistency regarding the survival benefit of ACT for rectal cancer. EORTC 22921 and PROCTORSCRIPT trials $[1,33]$ failed to show a survival benefit. On the other hand, an improvement in survival was reported in the QUASAR trial [34] and in the Cochrane Collaboration meta-analysis [35]. The National Comprehensive Cancer Network guidelines recommend ACT for patients with LARC [5]. The ESMO guidelines published in 2017 recommended ACT for a subgroup of patients with rectal cancer after any NCRT and surgery with the caution that the evidence to support this recommendation is weak [6]. Our study showed ACT to be a significant predictor of survival. This finding adds to the body of evidence in support of survival benefit from ACT.

In our study, ACT showed a survival benefit to the whole cohort while $\mathrm{pCR}$ was a significant predictor of survival independent of the use of ACT. Therefore, we expect the survival benefit from ACT to be less or even absent in those who achieved a pCR. Due to the limitation of the study size, this hypothesis could not be tested. Nevertheless, similar finding was reported in a meta-analysis by Maas et al. where ACT did not add to the survival of those with a pCR after NACRT [7]. 
Disease stage and status of the resection margin were predictors of survival, which is consistent with other studies [36,37]. We also found that females had better survival than males. This is consistent with the latest Surveillance, Epidemiology, and End Results (SEER) program report [38].

It is not clear to us why patients who reside in rural areas have a better survival compared with those who live in urban setting. Rural-living lifestyle is unlikely to contribute to this finding since the life expectancy at birth for rural Manitoba was lower than the national average in a report published in 2011 [39]. Unfortunately, we did not collect data on performance status, BMI or comorbidities at time of diagnosis, which may be confounding variables. Further research would be needed to verify and investigate this association.

Limitations of this study are its relatively small sample size (280 patients) and missing demographic and pathological information. Potential selection bias such as poor fitness, personal choice or other considerations might have contributed to the number of patients who did not receive ACT. Finally, this work represents a single institutional experience. Nevertheless, our results concur with previously published data especially from other centers in Canada [13].

\section{Conclusion}

Patients with LARC who achieved pCR after NACRT were less likely to have recurrence and had better survival. Thus, pCR should be considered as an endpoint for oncological outcome. Strategies to increase the chances of complete response by maximizing the neoadjuvant therapy should be explored.

ACT was associated with an improvement in survival. However, further analysis would be required to determine if patients who achieved pCR derive additional survival benefit from ACT.

\section{Future perspective}

Based on this study, we envisage clinical trials of escalating dose of neoadjuvant radiation to the maximum tolerated dose for patients with LARC. This will lead to more clinical and pathological complete responses. Consequently, a subgroup of patients might avoid surgery altogether or get a down-scaled surgical procedure to preserve anal sphincter. Patients and doctors will be more confident to omit ACT when pCR is achieved.

\section{Financial \& competing interests disclosure}

The authors have no relevant affiliations or financial involvement with any organization or entity with a financial interest in or financial conflict with the subject matter or materials discussed in the manuscript. This includes employment, consultancies, honoraria, stock ownership or options, expert testimony, grants or patents received or pending, or royalties.

No writing assistance was utilized in the production of this manuscript.

\section{Ethical conduct of research}

The authors state that they have obtained appropriate institutional review board approval or have followed the principles outlined in the Declaration of Helsinki for all human or animal experimental investigations.

\section{Open access}

This work is licensed under the Creative Commons Attribution4.0 License. To view a copy of this license, visit http://creativecommons.org/licenses/by/4.0/

\section{References}

Papers of special note have been highlighted as: $\bullet$ of interest; $\bullet \bullet$ of considerable interest

1. Bosset J-F, Collette L, Calais G et al. Chemotherapy with preoperative radiotherapy in rectal cancer. N. Engl. J. Med. 355(11), 1114-1123 (2006).

2. Sauer R, Becker H, Hohenberger W et al. Preoperative versus postoperative chemoradiotherapy for rectal cancer. N. Engl. J. Med. 351(17), 1731-1740 (2004).

3. Maas M, Nelemans PJ, Valentini V et al. Long-term outcome in patients with a pathological complete response after chemoradiation for rectal cancer: a pooled analysis of individual patient data. Lancet Oncol. 11(9), 835-844 (2010).

-. Pathological complete response ( $\mathrm{pCR}$ ) predicts better long-term outcome.

4. Bosset J-F, Calais G, Mineur L et al. Fluorouracil-based adjuvant chemotherapy after preoperative chemoradiotherapy in rectal cancer: long-term results of the EORTC 22921 randomised study. Lancet Oncol. 15(2), 184-190 (2014).

5. Rectal Cancer NCCN Clinical Practice Guidelines in Oncology ( $\mathrm{NCCN}^{\circledR}$ Guidelines). Version 3 (2017). www.nccn.org/professionals/physician_gls/PDF/rectal.pdf 
6. Glynne-Jones R, Wyrwicz L, Tiret E et al. Rectal cancer: ESMO Clinical Practice Guidelines for diagnosis, treatment and follow-up. Ann. Oncol. 28(Suppl. 4), IV22-IV40 (2017).

-. Lack of evidence leads to a weak recommendation for adjuvant chemotherapy (ACT) for rectal cancer.

7. Maas M, Nelemans PJ, Valentini V et al. Adjuvant chemotherapy in rectal cancer: defining subgroups who may benefit after neoadjuvant chemoradiation and resection: a pooled analysis of 3313 patients. Int. J. Cancer 137(1), 212-220 (2015).

- $\quad$ Patients with pCR after neoadjuvant chemoradiotherapy may not benefit from ACT.

8. Cancer in Manitoba. The Public Health Act (2006). http://web2.gov.mb.ca/laws/statutes/ccsm/p210e.php

9. Weir HK, Johnson CJ, Mariotto AB et al. Evaluation of North American Association of Central Cancer Registries' (NAACCR) data for use in population-based cancer survival studies. JNCI Monogr. 2014(49), 198-209 (2014).

10. Edge SB, Compton CC. The American Joint Committee on Cancer: the 7 th edition of the AJCC cancer staging manual and the future of TNM. Ann. Surg. Oncol. 17(6), 1471-1474 (2010).

11. Myerson RJ, Garofalo MC, El Naqa I et al. Elective clinical target volumes for conformal therapy in anorectal cancer: a radiation therapy oncology group consensus panel contouring atlas. Int. J. Radiat. Oncol. 74(3), 824-830 (2009).

12. Washington MK, Berlin J, Branton $\mathrm{P}$ et al. Protocol for the examination of specimens from patients with primary carcinoma of the colon and rectum. Arch. Pathol. Lab. Med. 133(10), 1539-51 (2009).

13. Armstrong D, Raissouni S, Price Hiller J et al. Predictors of pathologic complete response after neoadjuvant treatment for rectal cancer: a multicenter study. Clin. Colorectal Cancer 14(4), 291-295 (2015).

-. Canadian retrospective data showing PCR rate similar to ours.

14. Díaz-González JA, Calvo FA, Cortés J et al. Prognostic factors for disease-free survival in patients with T3-4 or N+ rectal cancer treated with preoperative chemoradiation therapy, surgery, and intraoperative irradiation. Int. J. Radiat. Oncol. 64(4), 1122-1128 (2006).

15. Habrgama A, Perez R, Nadalin W et al. Long-term results of preoperative chemoradiation for distal rectal cancer correlation between final stage and survival. J. Gastrointest. Surg. 9(1), 90-101 (2005).

16. Rodel C, Martus P, Papadoupolos T et al. Prognostic significance of tumor regression after preoperative chemoradiotherapy for rectal cancer. J. Clin. Oncol. 23(34), 8688-8696 (2005).

17. Valentini V, Coco C, Picciocchi A et al. Does downstaging predict improved outcome after preoperative chemoradiation for extraperitoneal locally advanced rectal cancer? A long-term analysis of 165 patients. Int. J. Radiat. Oncol. 53(3), 664-674 (2002).

18. Allegra CJ, Yothers G, O'Connell MJ et al. Neoadjuvant 5-FU or capecitabine plus radiation with or without oxaliplatin in rectal cancer patients: a Phase III randomized clinical trial. J. Natl. Cancer Inst. 107(11), djv248 (2015).

19. Aschele C, Cionini L, Lonardi S et al. Primary tumor response to preoperative chemoradiation with or without oxaliplatin in locally advanced rectal cancer: pathologic results of the STAR-01 randomized Phase III trial. J. Clin. Oncol. 29(20), 2773-2780 (2011).

20. Gérard J-P, Azria D, Gourgou-Bourgade S et al. Clinical outcome of the ACCORD 12/0405 PRODIGE 2 randomized trial in rectal cancer. J. Clin. Oncol. 30(36), 4558-65 (2012).

21. Rödel C, Graeven U, Fietkau R et al. Oxaliplatin added to fluorouracil-based preoperative chemoradiotherapy and postoperative chemotherapy of locally advanced rectal cancer (the German CAO/ARO/AIO-04 study): final results of the multicentre, open-label, randomised, Phase 3 trial. Lancet Oncol. 16(8), 979-989 (2015).

22. Benevento I, De Felice F, Musio D, Tombolini V. The addition of target therapy to neoadjuvant chemoradiotherapy in locally advanced rectal cancer: a review. Chemotherapy 62(5), 314-322 (2017).

23. Hall MD, Schultheiss TE, Smith DD, Fakih MG, Wong JYC, Chen Y-J. Effect of increasing radiation dose on pathologic complete response in rectal cancer patients treated with neoadjuvant chemoradiation therapy. Acta Oncol. 55(12), 1392-1399 (2016).

- $\quad$ Escalating radiation dose leads to a better tumor response.

24. Gérard J-P, Azria D, Gourgou-Bourgade S et al. Comparison of two neoadjuvant chemoradiotherapy regimens for locally advanced rectal cancer: results of the Phase III trial ACCORD 12/0405-Prodige 2. J. Clin. Oncol. 28(10), 1638-1644 (2010).

25. O'Connell MJ, Colangelo LH, Beart RW et al. Capecitabine and oxaliplatin in the preoperative multimodality treatment of rectal cancer: surgical end points from National Surgical Adjuvant Breast and Bowel Project trial R-04. J. Clin. Oncol. 32(18), 1927-1934 (2014).

26. Barresi V, Reggiani Bonetti L, Ieni A, Branca G, Tuccari G. Histologic prognostic markers in stage IIA colorectal cancer: a comparative study. Scand. J. Gastroenterol. 51(3), 314-320 (2016).

27. Nikberg M, Chabok A, Letocha H, Kindler C, Glimelius B, Smedh K. Lymphovascular and perineural invasion in stage II rectal cancer: a report from the Swedish colorectal cancer registry. Acta Oncol. 55(12), 1418-1424 (2016).

28. Yoon WH, Kim HJ, Kim CH, Joo JK, Kim YJ, Kim HR. Oncologic impact of pathologic response on clinical outcome after preoperative chemoradiotherapy in locally advanced rectal cancer. Ann. Surg. Treat. Res. 88(1), 15-20 (2015).

29. Jhaveri KS, Hosseini-Nik H, Thipphavong $S$ et al. MRI detection of extramural venous invasion in rectal cancer: correlation with histopathology using elastin stain. AJR Am. J. Roentgenol. 206(4), 747-755 (2016).

30. Smith NJ, Barbachano Y, Norman AR, Swift RI, Abulafi AM, Brown G. Prognostic significance of magnetic resonance imaging-detected extramural vascular invasion in rectal cancer. Br. J. Surg. 95(2), 229-236 (2008). 
31. Sauer R, Liersch T, Merkel S et al. Preoperative versus postoperative chemoradiotherapy for locally advanced rectal cancer: results of the German CAO/ARO/AIO-94 randomized Phase III trial after a median follow-up of 11 years. J. Clin. Oncol. 30(16), 1926-1933 (2012).

32. Helewa RM, Turner D, Wirtzfeld D et al. Geographical disparities of rectal cancer local recurrence and outcomes. Dis. Colon Rectum 56(7), 850-858 (2013).

- Survival in the same location (Manitoba, Canada) before the implementation of neoadjuvant chemoradiotherapy and use of ACT.

33. Breugom AJ, van Gijn W, Muller EW et al. Adjuvant chemotherapy for rectal cancer patients treated with preoperative (chemo)radiotherapy and total mesorectal excision: a Dutch Colorectal Cancer Group (DCCG) randomized Phase III trial. Ann. Oncol. 26(4), 696-701 (2015).

34. QUASAR Collaborative Group, Gray R, Barnwell J et al. Adjuvant chemotherapy versus observation in patients with colorectal cancer: a randomized study. Lancet 370(9604), 2020-2029 (2007).

35. Petersen SH, Harling H, Kirkeby LT, Wille-Jørgensen P, Mocellin S. Postoperative adjuvant chemotherapy in rectal cancer operated for cure. In: Cochrane Database of Systematic Reviews. Petersen SH (Ed). John Wiley \& Sons Ltd, Chichester, UK (2012).

36. Sung S, Kim SH, Lee JH et al. Continuous effect of radial resection margin on recurrence and survival in rectal cancer patients who receive preoperative chemoradiation and curative surgery: a multicenter retrospective analysis. Int. J. Radiat. Oncol. Biol. Phys. 98(3), 647-653 (2017).

37. Van Gijn W, Marijnen CAM, Nagtegaal ID et al. Preoperative radiotherapy combined with total mesorectal excision for resectable rectal cancer: 12-year follow-up of the multicentre, randomised controlled TME trial. Lancet Oncol. 12(6), 575-582 (2011).

38. SEER Cancer Stat Facts: Colon and Rectum Cancer. National Cancer Institute. Bethesda M. Cancer of the Colon and Rectum - Cancer Stat Facts. https://seer.cancer.gov/statfacts/html/colorect.html

39. Disparities in life expectancy. www.statcan.gc.ca/pub/82-624-x/2011001/article/11427-eng.htm\#a5 
